\title{
Predictors of discogenic pain in magnetic resonance imaging: a retrospective study of provocative discography performed by posterolateral approach
}

\author{
Anuj Jain ${ }^{1}$, Suruchi Jain ${ }^{2}$, Swapnil Kumar Barasker ${ }^{3}$, and Amit Agrawal ${ }^{4}$ \\ 'Department of Anesthesiology, All India Institute of Medical Sciences, Bhopal, India \\ ${ }^{2}$ Department of Nuclear Medicine, All India Institute of Medical Sciences, Bhopal, India \\ ${ }^{3}$ Department of Anesthesiology, Sri Aurobindo Medical College and Post Graduate Institute, Indore, India \\ ${ }^{4}$ Department of Neurosurgery, All India Institute of Medical Sciences, Bhopal, India
}

Received May 3, 2021

Revised July 1, 2021

Accepted July 5, 2021

Handling Editor: Francis S. Nahm

\section{Correspondence}

Swapnil Kumar Barasker

Department of Anesthesiology, Sri

Aurobindo Medical College and Post

Graduate Institute, Indore Ujjain State

Highway, Near MR10 Crossing, Indore

453111, India

Tel: +91-8109101245

Fax: +91-731-4231010

E-mail: swapnilkbarasker@gmail.com
Background: Provocative discography (PD) is a test that is useful in diagnosing discogenic pain (DP). In this study, to diagnose DP, we used a posterolateral approach of needle placement and followed pressure criteria laid down by the Spine Intervention Society. The aim was to identify the correlation between magnetic resonance imaging (MRI) findings (desiccation, high intensity zone and change in shape and size of the disc) and the results of PD.

Methods: Records of 50 patients who underwent PD for DP were analyzed. A total of 109 PDs were performed, with 54 suspect and 55 control discs. Alternate pain generators were ruled out.

Results: A total of 35 suspect discs were positive on PD. The mean disc pressure in the suspect disc was $31.9 \pm 7.9$ psi (range, 15-44). Of the 50 patients who underwent PD, 35 had positive MRI findings. A significant positive correlation was found only between disc desiccation and discography result $(r=0.6, P<0.001)$. Logistic regression analysis revealed that only desiccation successfully predicted the result of discography $(\mathrm{OR}=26.5, P<0.001)$; a high intensity zone and a disc protrusion/ extrusion had an OR 2.3 and 1.24, respectively. Disc desiccation of Pfirmann grade 3 or more had a sensitivity and specificity of 0.93 and 0.64 respectively in identifying painful discs; the positive likelihood ratio was 2.58 while the negative likelihood ratio was 0.11 .

Conclusions: In patients with DP, disc desiccation is the most useful MRI feature that predicts a painful disc on PD.

Key Words: Desiccation; Diagnosis; Discography; Intervertebral Disc; Intervertebral Disc Degeneration; Low Back Pain; Magnetic Resonance Imaging; Pain Management; Sensitivity and Specificity; Spinal Diseases.

\section{INTRODUCTION}

Discogenic pain (DP) is an important etiology of low back pain (LBP). Diagnosis and management of DP is a debat- able topic among physicians who treat LBP [1-4]. Unlike magnetic resonance imaging (MRI), which gives anatomic details, provocative discography (PD) is a physiological test to specifically identify a painful disc. PD is a diagnos- (c) This is an open-access article distributed under the terms of the Creative Commons Attribution Non-Commercial License (http://creativecommons.org/licenses/by-nc/4.0/), which permits unrestricted non-commercial use, distribution, and reproduction in any medium, provided the original work is properly cited.

(c) The Korean Pain Society, 2021
Author contributions: Anuj Jain: Study conception; Suruchi Jain: Writing/ manuscript preparation; Swapnil Kumar Barasker: Writing/manuscript preparation; Amit Agrawal: Methodology. 

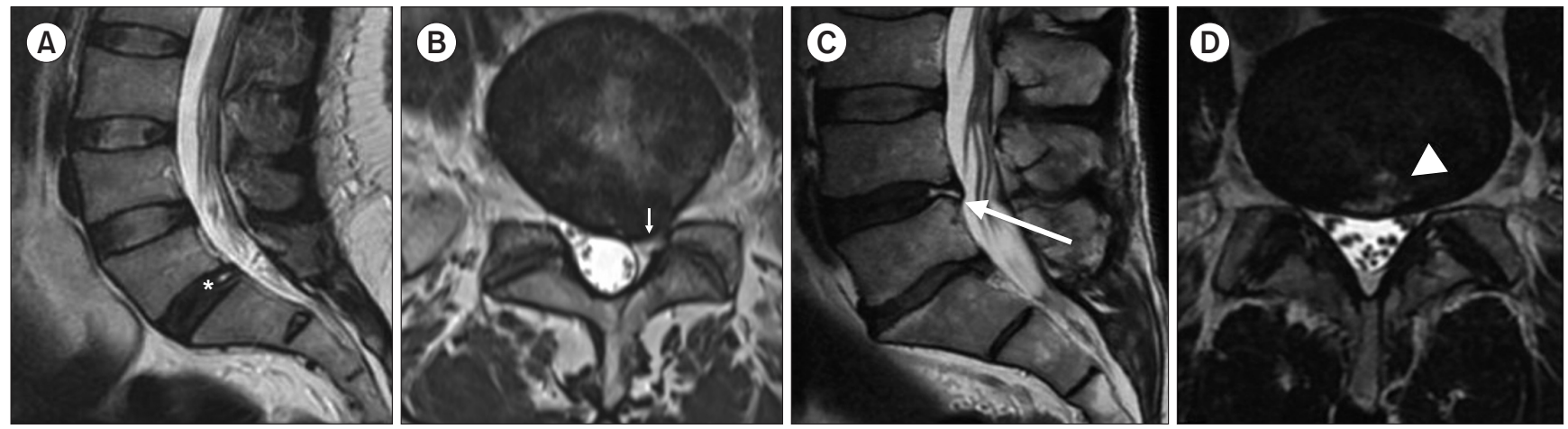

Fig. 1. Lumbar spine magnetic resonance imaging (MRI) images showing desiccation, high intensity zone (HIZ), and protrusion/extrusion. (A) Sagittal T2-weighted MRI image showing a HIZ and Pfirrmann grade IV. ' *' denotes the HIZ. (B) Axial T2-weighted MRI image showing a left paracentral disc protrusion (arrow). (C) Sagittal T2- weighted MRI image showing HIZ and Pfirrmann grade IV disc (arrow). (D) Axial T2- weighted MRI image showing a HIZ without protrusion/extrusion (arrowhead).

tic and prognostic test for DP, but, over time, the popularity of PD has decreased, possibly due to a poor correlation between the results of PD and results of spine fixation surgeries (SFS) [5]. The main reason for this discord seems to be non-uniform technique and classification criteria [6] for a painful disc on PD. Most of the publications are ambiguous about the technical aspects of PD [6]. Although the Spine Intervention Society (SIS) has laid down standard criteria for interpreting the results, they are seldom implemented.

In this study, we used PD to identify painful disc in patients who had chronic LBP with a suspicion of DP. To pressurize the posterior part of the disc, we adopted a posterolateral approach for needle placement. The pressure criteria laid down by the SIS were implemented to interpret the results. The aim of this study was to identify the correlation between MRI findings (desiccation, high intensity zone [HIZ], and change in the shape and size of the disc) and the results of PD.

\section{MATERIALS AND METHODS}

In the current retrospective study, exemption was obtained from the institutional review board (IHECLOP/2019/ IM0237). Patients visiting the pain clinic between July 2018 and December 2019 were screened and the records of 576 patients with LBP were analyzed. PD was done in patients who, based on history/examination and radiological findings, had a high index of clinical suspicion for DP; other etiologies of LBP were ruled out. Clinical features considered suggestive of DP were severe episodic axial LBP, increased on prolonged sitting or forward bending and showing centralization on Mckenzie exercises $[7,8]$. An MRI feature suggestive of DP was a desiccated disc alone (Pfirrman grade 3 or more) [9], or in combination with protrusion/extrusion or a HIZ (Fig. 1).

Patients who had advanced spondylitis changes, spondylolisthesis, multiple level prolapsed intervertebral disc, previous spine surgery, collagen vascular disorders, mental health issues, or any sensori-motor deficit were not subjected to PD. Before subjecting the patients to PD, alternate pain generators like facet joint, sacroiliac joints, and myofascial components were ruled out by means of targeted diagnostic pain relieving interventions.

All the PDs were performed under strict asepsis and fluoroscopic guidance. A single shot of intravenous antibiotic was given 30 minutes before the procedure. Intraoperative monitoring was done using electrocardiogram, pulseoximetry, and blood pressure monitoring.

With the patient placed in prone position with a pillow under the belly, PD was performed using the posterolateral approach, as described, for disc access in percutaneous transforaminal endoscopic discectomy (Figs. 2, 3). A 22G, $20 \mathrm{~cm}$ Quincke needle was used to access the disc. The suspected disc was pressurized manually by injecting contrast medium in aliquots of $0.5 \mathrm{~mL}$, and the patients' response to provocation was noticed; categorization of the results was done as per the SIS criteria [10]. The disc was classified as painful if, upon injection of diluted radiocontrast (Omnipaque; GE Healthcare, Bengaluru, India), 1:1 in normal saline, the patient experienced concordant pain that was greater than 6 on the verbal numerical rating scale (NRS) or had more than $70 \%$ pain reproduction, at a pressure of less than 50 psi above the opening pressure, with a painless adjacent control disc; no more than $3 \mathrm{~mL}$ of contrast medium was injected. Opening pressure was defined as the pressure when the contrast media was first visualized at the tip of the needle by fluoroscopy. The control disc was an adjacent radiologically normal disc. Discography was considered negative if $3 \mathrm{~mL}$ of contrast medium was injected and still the pressure in the disc 
did not reach 50 psi or the elicited pain was discordant in nature. A disc-monitor discography probe (Stryker, Kalamazoo, MI) was used for pressure measurements during PD. A disc-monitor helps to standardize PD by providing and recording the opening pressure, maximum pressure achieved, and volume injected [11].

Based on the formula for sample size calculation by Green [12] $(\mathrm{N}=104+\mathrm{X}$, where $\mathrm{N}$ is the sample size and $\mathrm{X}$ is the number of independent variable).We considered age, containment of the disc, disc desiccation, HIZs, and protrusion/ extrusion as the independent variables. Hence, a total of 109 PDs were needed for analysis. The power of study was kept at 80 percent.

\section{Statistical analysis}

Statistical analysis was done using the SPSS ver. 20.0 (IBM Co., Armonk, NY) software. Quantitative data like age, body mass index (BMI), NRS, and mean disc pressure were

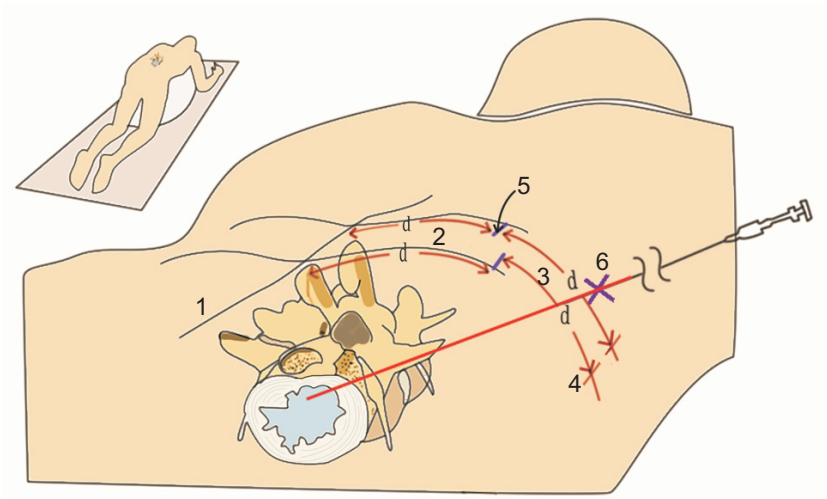

Fig. 2. Diagrammatic explanation of the surface marking used for identifying the site of needle insertion; also depicted is the needle trajectory. 1: line in the midline along the spinous processes, 2 : line along the intervertebral disc with C-arm in anteroposterior view, 3: line along the intervertebral disc with the $\mathrm{C}$-arm in the lateral view, 4 : line denoting center of the disc with $\mathrm{C}$-arm in the lateral view, 5: a perpendicular from line 2 onto line 3 at a distance ' $d$ ' from the midline, 6 : point of needle insertion for posterolateral approach of provocative discography, d: distance from skin surface to line ' 4 ' as measure in lateral view of C-arm. expressed as mean \pm standard deviation, and were analysed using independent sample Student's $t$-test. Categorical variables were expressed as counts (percentage) and were analysed using a chi-square test, Wilcoxon's $U$-test, or Fisher's exact test.

An adjusted multivariate logistic regression model analysis was performed for identifying the association of MRI findings and PD results. Results were presented as odds ratio (OR) with $95 \%$ confidence interval ( $95 \% \mathrm{CI})$. All statistical assessments were 2 tailed and significance was set at a $P$ value less than 0.05 .

\section{RESULTS}

After analyzing records of 576 patients with chronic LBP, 526 were excluded, and 50 patients that had undergone PD were studied; 109 PDs were performed, with 54 suspected discs and 55 control discs (Fig. 4). The mean age $45.3 \pm$ 6.7 years, $54 \%$ patients were male, mean BMI was $26.7 \pm$ 2.3 , and the mean duration of symptoms was $11.27 \pm 4.0$ months. The mean pain score before intervention on the NRS was $5.7 \pm 1.8$. Forty discs were painful on discography, including 35 suspected and 5 control discs. Eighty percent of the suspected discs were at L4-5 level, while the rest were at L5-S1 level. For suspected discs at the L4-5 level, the L3-4 disc acted as the control, while for those at the L5S1 level, the L4-5 was the control.

The mean disc pressure in the painful discs was $31.9 \pm 7.9$ psi (range, 15-44 psi), with less than 20 psi in 2, 20-30 psi in $10,30-40$ psi in 15 , and $40-50$ psi in 8 patients. Out of the 50 patients who underwent $\mathrm{PD}, 35$ had positive MRI findings. Thirty-nine suspect discs were contained (no spillage of contrast in the extra discal space). A significant positive correlation was found only between disc desiccation and the results of PD (Table 1).

An adjusted logistic regression analysis revealed that only disc desiccation successfully predicted the result of discography ( $P<0.001$; OR, 26.5; 95\% CI, 4.5-155.6). Sixtysix percent of discs having HIZs were painful, and $65 \%$ discs with protrusion/extrusions were painful, but neither
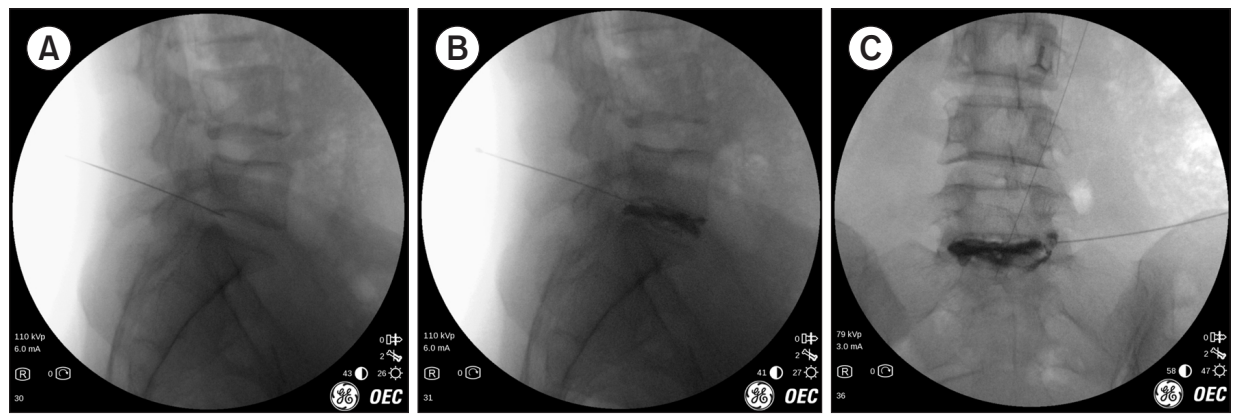

Fig. 3. Fluroscopic images of provocative discography. (A) Lateral view with needle in disc space. (B) Disc with injected dilute radiocontrast (Omnipaque, 1:1 in normal saline) in lateral view. (C) Disc with injected dilute radiocontrast (Omnipaque, 1:1 in normal saline) in antero-posterior view. 


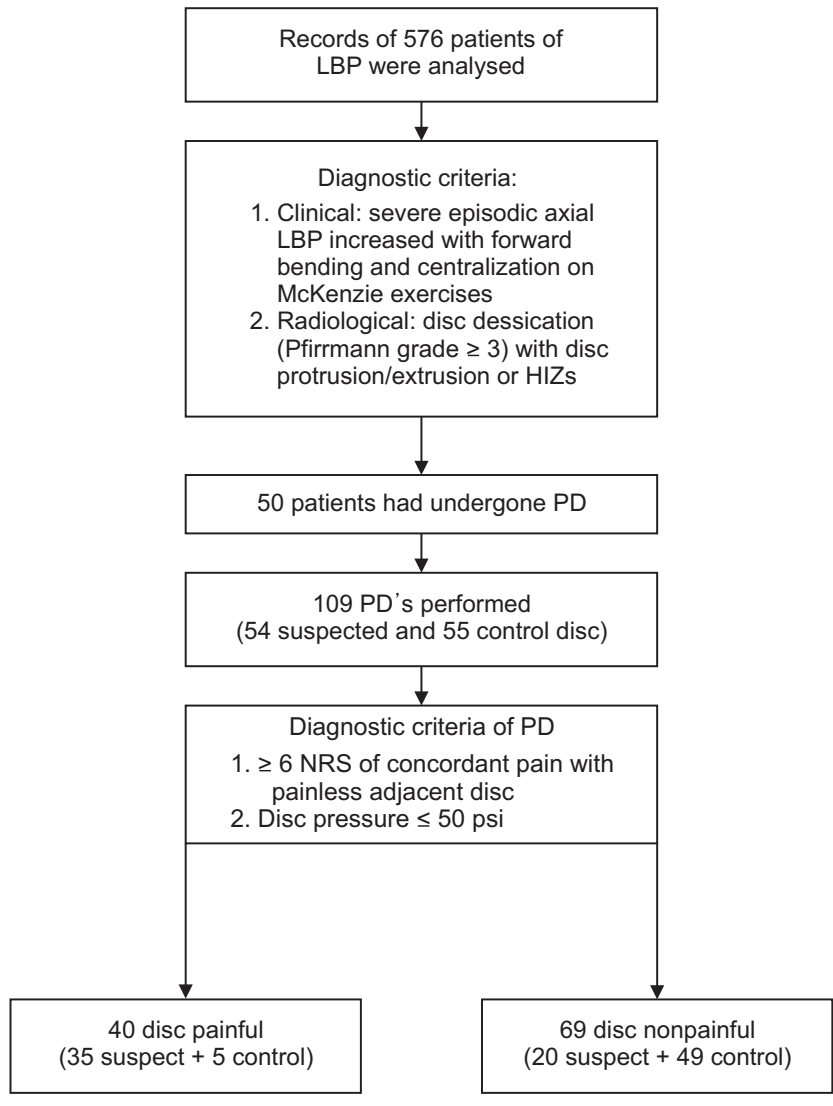

Fig. 4. Study summary. LBP: low back pain, PD: provocative discography, NRS: numerical rating scale, psi: per square inch, HIZ: high intensity zone.

HIZ nor protrusion/extrusion had a statically significant OR $(P=0.330$ [OR, 2.3; 95\% CI, 0.4-12.5], and $P=0.800$ [OR, 1.24; 95\% CI, 0.28-6.8], respectively). The regression model had an overall correct prediction rate of $82 \%$.

A receiver operating characteristic curve was generated for the result of PD and disc desiccation, the area under the curve was 0.78 (95\% CI, 0.64-0.93; $P=0.001$ ), and the sensitivity of disc desiccation in predicting the results of PD was 0.93 , while the specificity was 0.64 . The positive likelihood ratio (LR+) was 2.58 and the negative likelihood ratio (LR-) was 0.11 (Fig. 5). Apart from a transient increase in the intensity of back ache, no other serious side-effects were observed.

\section{DISCUSSION}

PD has been controversial and recently its utility has been questioned. Non-uniform technique and diagnostic criteria are possibly the main reason why the results of PD are difficult to interpret clinically [13]. PD has been demonstrated to have a degenerative effect on intervertebral discs [14]. The study was done in the 1990s, had a small
Table 1. Spearman's correlation coefficient of study parameters and pain on discography

\begin{tabular}{lcc}
\hline \multicolumn{1}{c}{ Parameter } & $\begin{array}{c}\text { Spearman's } \\
\text { coefficient }\end{array}$ & $P$ value \\
\hline Age & -0.31 & $0.027^{*}$ \\
Containment status of the disc & 0.07 & 0.600 \\
Desiccation of the disc & 0.61 & $<0.001^{*}$ \\
Presence of HIZ & 0.16 & 0.270 \\
Nucleus prolapse (protrusion/extrusion) & 0.06 & 0.660 \\
\hline
\end{tabular}

HIZ: high intensity zone.

${ }^{*} P$ value less than 0.05 is significant.

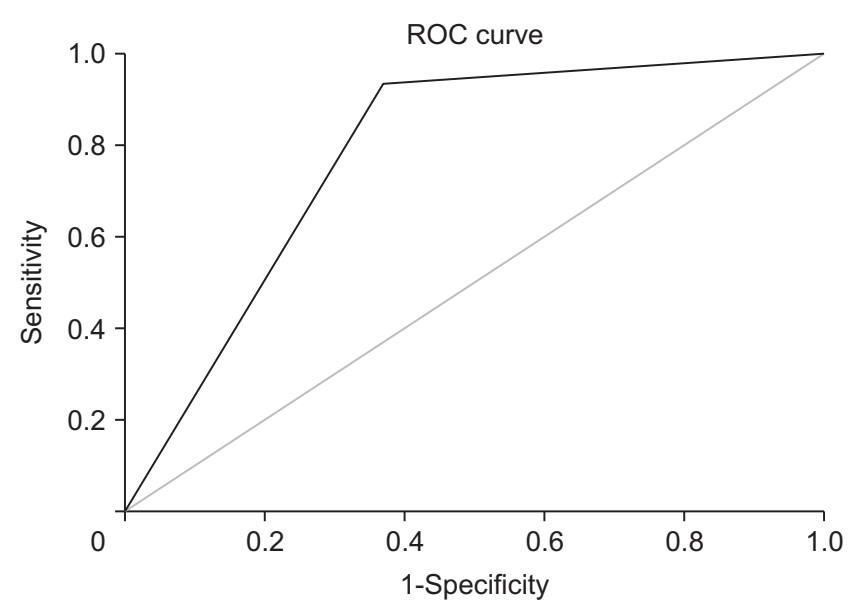

Fig. 5. Receiver operating characteristic curve for disc desiccation and painful disc.

sample size, and did not adhere to the SIS guidelines for PD. They used extremely high pressures (100 psi), and the accelerated degeneration could have been due to the exposure of the annulus to very high intradiscal pressure $[15,16]$. Another small study $(n=36)$ of matched control demonstrated no worsening of symptoms or radiological grade of disc degeneration at the 5-year follow-up [17].

With time, PD has also evolved, with the maximum pressure allowed now being much less (50 psi). Needles of a much smaller caliber are also now used, thereby minimizing the damage to the annulus [18]. In this study we implemented a pressure controlled protocol of PD. One important observation of this study is the provocation of concordant pain at a pressure lower than the recommended upper limit. Placement of needle tip in the posterior part rather than the center of disc, and hence nearer to the site of maximum disc damage, seems to be the reason for this observation. This small change in the technique not only increases the clinical relevance of the procedure but also theoretically reduces the chances of disc damage over a period of time.

MRI criteria for diagnosis of a painful disc have been 
controversial; most of the controversy is due to nonuniformity of patient selection, diagnosis, and procedure related criterias. The sensitivity, specificity, positive predictive value (PPV), and negative predictive value of T2weighted images in detecting the symptomatic disc have been estimated to be $94 \%, 71 \%, 59 \%$, and $97 \%$, respectively [19]. An MRI study on healthy volunteers revealed disc herniation and extrusion in $7 \%$ and $13 \%$ cases, respectively [20].

High HIZs, disc desiccation, disc enlargement, and reduction in disc height are the most common MRI criteria considered significant by radiologists. Many consider HIZ to be the most relevant feature in a T2W MRI, however the diversity in results in intriguing. A significant proportion of discs having HIZs are painless on PD, and the two have been demonstrated not to have any significant correlation [21-24]. HIZs demonstrated on an axial loaded MRI did not show a significant correlation with a painful disc [25]. On the contrary, HIZs in morphologically abnormal discs (Dallas grades 3, 4, and 5) have been demonstrated to have a significant correlation with concordant pain reproduction $(P<0.001)$. The sensitivity, specificity, and PPV of HIZs in isolation or in combination with other radiological features ranges from $45.5 \%-81 \%, 69 \%-97.8 \%$, and $39 \%-87 \%$, respectively [25-27]. HIZ in conjunction with endplate changes can have a sensitivity and specificity of 94 and $77 \%$, respectively [5]. In the current study, we did not find HIZs to predict the results of PD.

A change in the shape and size of the disc has also been considered an important MRI criteria. Disc bulge has been found not to correlate with disc pain [28]. Disc protrusion has been demonstrated to have a sensitivity of $68.2 \%$, specificity of $80.6 \%$, PPV of $53.6 \%$ [27], and the PPV of disc extrusion has been found to be $93 \%$ [28]. Another criterion considered important in lumbosacral MRI is disc desiccation [29]. Isolated disc desiccation at the L4-5 level may be seen in Bertolotti syndrome [30]. O'Neil et al. [29] demonstrated that a moderate loss of nuclear signal is the most important factor that predicts a positive discography and, along with disc bulge, has the best combination of sensitivity $(79.8 \%)$ and specificity (79.3\%). They concluded that normal or severe loss of signal intensity in the disc is most likely to be painless and, combining other MRI parameters, has no influence on test performance [29]. Although a contrarian view is also available for this parameter [27], our results are in congruence with this observation.

In this study, we carefully selected the patients (based on high probability clinical features and high probability MRI findings), implemented a pressure-controlled protocol of $\mathrm{PD}$, and also stimulated the posterior part of the disc, thereby removing most of the confounding factors that impaired the quality of previous research. In an experimental study in pigs, injection of contrast medium into one disc led to a $16 \%$ (3.2-37.0) increase in intradiscal pressure of the adjacent non-injected disc; this observation is important, as it may increase the false positive results of PD [31]. Although this aspect of PD has not been studied in humans, our approach of posterolateral discography can theoretically reduce this problem by utilizing lower intradiscal pressure for diagnosis of DP.

Based on a meta-analysis, if the SIS criteria are followed $[10,16], \mathrm{PD}$ has a low false positive rate of $6 \%$ per disc [13,32]; however, given the variability of techniques this incidence may be much higher. In order to address this problem of false positive results, a few investigators have suggested an alternative approach, i.e., analgesic discography/discoblock $[33,34]$. In discoblock, in contrast to PD, a small amount of local anesthetic is injected into the suspected disc and relief in back ache is assessed. If a patient's pain is relieved after discoblock, then that disc is considered to be the pain generator. An analgesic discography can further solve the problem of false positives. A study with a small study group reported that, at a 3 year follow-up, results of surgery-based discoblock (analgesic discography) were better than those based on PD [35].

Based on the finding in our study, we recommend the use of MRI as a screening tool for chronic LBP and utilization of a pressure-controlled PD in selecting patients for SFS. The findings of this study suggest that over and above clinical features, MRI provides useful information in identifying DP. In MRI, disc desiccation is the only important finding as far as prediction of a painful disc is concerned. In the current study, we adopted a posterolateral approach for needle placement, avoiding puncture of the thecal sac [36], and thereby pressurizing the posterior third of the disc. Stimulation of the posterior part of disc makes the results of this study more relevant from the clinical point of view, as these are the sites where the sinuvertebral nerve is mainly located and sensitized [37,38].

The present study has many limitations. The retrospective nature of the study is the biggest limitation. A larger sample size could have increased the power of study. Although, our data clinically address the usefulness of PD in decision-making for spinal operations, it lacks the corroborative data on the outcome of PD in SFS. Besides, it is data from a single center, so selection bias cannot be ruled out. A prospective randomized study, sequentially using a posterolateral approach for discography along with discoblock can give promising results as far as diagnosing DP is concerned.

In patients with DP, disc desiccation is the most useful MRI feature that predicts a painful disc on PD, with an OR of 26. The sensitivity and specificity of disc desiccation on MRI in predicting a painful disc on PD were 93 and 64, 
respectively, and the positive likelihood ratio and negative likelihood ratio were 2.58 and 0.11 , respectively.

\section{CONFLICT OF INTEREST}

No potential conflict of interest relevant to this article was reported.

\section{FUNDING}

No funding to declare.

\section{ORCID}

Anuj Jain, https://orcid.org/0000-0002-2105-5524

Suruchi Jain, https://orcid.org/0000-0002-3919-4581

Swapnil Kumar Barasker, https://orcid.org/0000-0002-3178-0623

Amit Agrawal, https://orcid.org/0000-0002-3287-5448

\section{REFERENCES}

1. Nachemson A, Zdeblick TA, O’Brien JP. Lumbar disc disease with discogenic pain. What surgical treatment is most effective? Spine (Phila Pa 1976) 1996; 21: 1835-8.

2. Zhou ZJ, Zhao FD, Fang XQ, Zhao X, Fan SW. Meta-analysis of instrumented posterior interbody fusion versus instrumented posterolateral fusion in the lumbar spine. J Neurosurg Spine 2011; 15: 295-310.

3. Robaina-Padrón FJ. [Controversies about instrumented surgery and pain relief in degenerative lumbar spine pain. Results of scientific evidence]. Neurocirugia (Astur) 2007; 18: 406-13. Spanish.

4. Mayer HM. [Discogenic low back pain and degenerative lumbar spinal stenosis - how appropriate is surgical treatment?]. Schmerz 2001; 15: 484-91. German.

5. Lei D, Rege A, Koti M, Smith FW, Wardlaw D. Painful disc lesion: can modern biplanar magnetic resonance imaging replace discography? J Spinal Disord Tech 2008; 21: 430-5.

6. Manchikanti L, Soin A, Benyamin RM, Singh V, Falco FJ, Calodney AK, et al. An update of the systematic appraisal of the accuracy and utility of discography in chronic spinal pain. Pain Physician 2018; 21: 91-110.

7. Levi D, Carnahan D, Horn S, Levin J. Is a history of severe episodic low back pain an indicator of a discogenic etiology? Pain Med 2018; 19: 1334-9.

8. Hancock MJ, Maher CG, Latimer J, Spindler MF, McAuley JH, Laslett M, et al. Systematic review of tests to identify the disc, SIJ or facet joint as the source of low back pain. Eur Spine J
2007; 16: 1539-50.

9. Pfirrmann CW, Metzdorf A, Zanetti M, Hodler J, Boos N. Magnetic resonance classification of lumbar intervertebral disc degeneration. Spine (Phila Pa 1976) 2001; 26: 1873-8.

10. Manchikanti L, Glaser SE, Wolfer L, Derby R, Cohen SP. Systematic review of lumbar discography as a diagnostic test for chronic low back pain. Pain Physician 2009; 12: 541-59.

11. Filippiadis DK, Mazioti A, Papakonstantinou O, Brountzos E, Gouliamos A, Kelekis N, et al. Quantitative discomanometry: correlation of intradiscal pressure values to pain reduction in patients with intervertebral disc herniation treated with percutaneous, minimally invasive, image-guided techniques. Cardiovasc Intervent Radiol 2012; 35: 1145-53.

12. Green SB. How many subjects does it take to do a regression analysis. Multivariate Behav Res 1991; 26: 499-510.

13. Stout A. Discography. Phys Med Rehabil Clin N Am 2010; 21: 859-67.

14. Cuellar JM, Stauff MP, Herzog RJ, Carrino JA, Baker GA, Carragee EJ. Does provocative discography cause clinically important injury to the lumbar intervertebral disc? A 10-year matched cohort study. Spine J 2016; 16: 273-80.

15. Koetsier E, Kallewaard JW, Maino P. Commentary on: does provocative discography cause clinically important injury to the lumbar intervertebral disc? A 10-year matched cohort study, by Cueller et al., Spine J 2016. Spine J 2017; 17: 610-1.

16. McCormick ZL, DeFrancesch F, Loomba V, Moradian M, Bathina R, Rappard G. Diagnostic value, prognostic value, and safety of provocation discography. Pain Med 2018; 19: 3-8.

17. Ohtori S, Inoue G, Orita S, Eguchi Y, Ochiai N, Kishida S, et al. No acceleration of intervertebral disc degeneration after a single injection of bupivacaine in young age group with follow-up of 5 years. Asian Spine J 2013; 7: 212-7.

18. Engel AJ, Stojanovic MP, Vorobeychik Y, MacVicar J. Highpressure discography may injure discs. Spine J 2017; 17: 6123.

19. Yoshida H, Fujiwara A, Tamai K, Kobayashi N, Saiki K, Saotome K. Diagnosis of symptomatic disc by magnetic resonance imaging: T2-weighted and gadolinium-DTPA-enhanced T1-weighted magnetic resonance imaging. J Spinal Disord Tech 2002; 15: 193-8.

20. Boos N, Rieder R, Schade V, Spratt KF, Semmer N, Aebi M. 1995 Volvo Award in clinical sciences. The diagnostic accuracy of magnetic resonance imaging, work perception, and psychosocial factors in identifying symptomatic disc herniations. Spine (Phila Pa 1976) 1995; 20: 2613-25.

21. Fang C, Zhang W, Chen L, Li H. The correlation between the high-intensity zone on a T2-weighted MRI and positive outcomes of discography: a meta-analysis. J Orthop Surg Res 2017; 12: 26.

22. Wang H, Li Z, Zhang C, Zhang W, Li L, Guo J, et al. Correlation between high-intensity zone on MRI and discography in 
patients with low back pain. Medicine (Baltimore) 2017; 96: e7222.

23. Carragee EJ, Paragioudakis SJ, Khurana S. 2000 Volvo Award winner in clinical studies: lumbar high-intensity zone and discography in subjects without low back problems. Spine (Phila Pa 1976) 2000; 25: 2987-92.

24. Wang ZX, Hu YG. Factors associated with lumbar disc highintensity zone (HIZ) on T2-weighted magnetic resonance image: a retrospective study of 3185 discs in 637 patients. J Orthop Surg Res 2018; 13: 307.

25. Hebelka H, Hansson T. HIZ's relation to axial load and low back pain: investigated with axial loaded MRI and pressure controlled discography. Eur Spine J 2013; 22: 734-9.

26. Lam KS, Carlin D, Mulholland RC. Lumbar disc high-intensity zone: the value and significance of provocative discography in the determination of the discogenic pain source. Eur Spine J 2000; 9: 36-41.

27. Kang CH, Kim YH, Lee SH, Derby R, Kim JH, Chung KB, et al. Can magnetic resonance imaging accurately predict concordant pain provocation during provocative disc injection? Skeletal Radiol 2009; 38: 877-85.

28. Chelala L, Trent G, Waldrop G, Dagher AP, Reinig JW. Positive predictive values of lumbar spine magnetic resonance imaging findings for provocative discography. J Comput Assist Tomogr 2019; 43: 568-71.

29. O'Neill C, Kurgansky M, Kaiser J, Lau W. Accuracy of MRI for diagnosis of discogenic pain. Pain Physician 2008; 11: 311-26.

30. Jain A, Agarwal A, Jain S, Shamshery C. Bertolotti syndrome: a diagnostic and management dilemma for pain physicians. Korean J Pain 2013; 26: 368-73.
31. Hebelka H, Gaulitz A, Nilsson A, Holm S, Hansson T. The transfer of disc pressure to adjacent discs in discography: a specificity problem? Spine (Phila Pa 1976) 2010; 35: E1025-9.

32. Wolfer LR, Derby R, Lee JE, Lee SH. Systematic review of lumbar provocation discography in asymptomatic subjects with a meta-analysis of false-positive rates. Pain Physician 2008; 11: 513-38.

33. Yu Y, Liu W, Song D, Guo Q, Jia L. Diagnosis of discogenic low back pain in patients with probable symptoms but negative discography. Arch Orthop Trauma Surg 2012; 132: 627-32.

34. Eck JC, Sharan A, Resnick DK, Watters WC 3rd, Ghogawala $\mathrm{Z}$, Dailey AT, et al. Guideline update for the performance of fusion procedures for degenerative disease of the lumbar spine. Part 6: discography for patient selection. J Neurosurg Spine 2014; 21: 37-41.

35. Ohtori S, Kinoshita T, Yamashita M, Inoue G, Yamauchi K, Koshi T, et al. Results of surgery for discogenic low back pain: a randomized study using discography versus discoblock for diagnosis. Spine (Phila Pa 1976) 2009; 34: 1345-8.

36. Peh W. Provocative discography: current status. Biomed Imaging Interv J 2005; 1: e2.

37. Kim HS, Paudel B, Chung SK, Jang JS, Oh SH, Jang IT. Transforaminal epiduroscopic laser ablation of sinuvertebral nerve in patients with chronic diskogenic back pain: technical note and preliminary result. J Neurol Surg A Cent Eur Neurosurg 2017; 78: 529-34.

38. Bogduk N, Tynan W, Wilson AS. The nerve supply to the human lumbar intervertebral discs. J Anat 1981; 132(Pt 1): 3956 . 